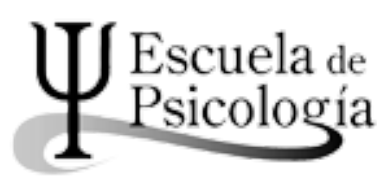

Wímblu, Rev. estudiantes Esc. de psicología, Univ. de Costa Rica. 12 (1): 9-24, 2017 / ISSN: 1659-2107

\title{
Tratamiento de relajación con biofeedback para la disminución de la frecuencia de los tics en un estudiante universitario con Síndrome de Tourette
}

Relaxation treatment with boifeedback for reduction of the frequency of tics in a university student with Tourette Syndrome

Catalina Alvarado Hidalgo $^{a}$
Valeria Angulo Castro $^{b}$
Julianne Beirute Herrera $^{c}$
Ariana Luna Aguilar $^{d}$
Isabel Molina Mesén $^{e}$

Resumen: El presente artículo consiste en un tratamiento de relajación con equipo de biofeedback que tiene como objetivo la reducción de los tics en un estudiante universitario con el Sndrome deTourette. Dicho padecimiento es un desorden neuropsiquiátrico que presenta movimientos espontáneos y repetitivos conocidos como tics. La relajación se induce por medio de respiración diafragmática y con el método conocido como relajación muscular progresiva a lo largo de 5 sesiones. Se logra reducir el número de tics en más de un $50 \%$ para la sexta sesión.

Palabras Clave: Tourette, equipo de retroalimentación, respiración, tics, relajación muscular progresiva.

Abstract: This article presents a relaxing treatment with biofeedback equipment that aims to reduce tics in a college student with Tourette Syndrome. This syndrome is a neuropsychiatric disorder which presents spontaneous and repetitive movements known as tics. The relaxation is induced by diaphragmatic breathing and the method known as progressive muscular relaxation. This relaxation was able to reduce the number of tics in more than $50 \%$ for the last session.

Key Words: tourette, biofeedback, breathing, tics, progressive muscular relaxation.

a Universidad de Costa Rica. Estudiante de psicología. Costa Rica. Correo electrónico: cata. alvarado06@gmail.com

b Universidad de Costa Rica. Bachiller en psicología. Costa Rica.

Correo electrónico: vale.angulo22@gmail.com

c Universidad de Costa Rica. Bachiller en psicología. Costa Rica.

Correo electrónico: beirutej@gmail.com

d Universidad de Costa Rica. Estudiante de psicología. Costa Rica.

Correo electrónico: ariana.m.I.a@gmail.com

e Universidad de Costa Rica. Bachiller en psicología. Costa Rica.

Correo electrónico: isabelmm18@gmail.com

** Recepción: 30/2/2016 Aceptación: 12/6/2016 


\section{Introducción}

El Síndrome de Tourette (TS) es un desorden neuropsiquátrico que se caracteriza por la persistencia de tics, es decir por movimientos espontáneos, repetitivos y estereotipados, ya sea verbales y/o motores (Ganos, Roessner \& Münchau, 2013; Carlson, 2014; Crossley, Seri, Stern, Robertson \& Cavanna 2014). Posee este nombre porque en 1880 , Georges Gilles de la Tourette fue la primera persona en publicar e investigar sobre el trastorno.

El síndrome es diagnosticado cuando la persona presenta múltiples tics motores y al menos uno verbal por más de un año, agravando su curso durante dicho tiempo (Asociación Americana de Psiquiatría, 2013; Busea, Schoenefeld Münchau \& Roessner, 2013). Entre los principales tics verbales se encuentran: muecas faciales, ladrar, toser y repetir palabras, en especial las vulgares (Carlson, 2014); y entre los motores: volver los ojos y sacudir la cabeza (Ganos et al., 2013).

Según Ganos et al. (2013), los tics se dividen en dos categorías, simples y complejos. Los simples son aquellos que incluyen solamente un músculo o un grupo de músculos; los complejos por su parte son más elaborados, repetitivos y pueden incluir diferentes partes del cuerpo.

Los tics, según Frank \& Eugenio (2013) son una respuesta del aumento de la tensión, que se da ante ciertos estímulos sensoriales específicos. Con base en esto se ha comprobado que en momentos de tensión y ansiedad los tics aumentan, mientras que en estados de relajación tanto los tics, como la tasa cardíaca y la tasa respiratoria disminuyen (Conelea, Woods \& Brandt, 2011; Felling \& Singer 2011; Busea, Schoenefelda, Münchaub \& Roessnera, 2013; Frank \& Cavanna, 2013; Neuner, et al. 2014).

Por otro lado, Frank \& Eugenio (2013) afirman que la excitación simpática, afecta la frecuencia de los tics en pacientes con síndrome de Tourette. También, el paso de los años parece tener un impacto, pues es alrededor de los 6 años de edad que comienza el síndrome y a partir de ese momento su severidad aumenta con el tiempo (Crossley et al., 2014).

Por lo mencionado y por la complejidad del padecimiento, no se posee un consenso sobre las bases biológicas del padecimiento ni tampoco sobre las causas de este, a pesar de que se conoce que la herencia tiene un papel fundamental, se desconoce cuáles genes específicamente lo causan. Sin embargo está establecido que se trata de una genética compleja que incluye extrañas mutaciones y factores epigenéticos y ambientales (Felling \& Singer, 2011; Longo, Fauci, Kasper, Hauser, Jameson \& Loscalzo, 2011; Buse et al., 2013; Denys et al., 2013; Serajee \& Mahbubu, 2015).

Por todo esto se han planteado diferentes teorías como la dopaminérgica y neuroanatómica (Mermillond et al., 2011), la cual establece que en las personas con TS hay alteraciones en la neurotransmisión de dopamina, específicamente en la unión de los receptores de D2 con la dopamina en el núcleo caudado (Bloch \&
Leckman, 2008; Longo et al., 2011; Greenberg, Aminoff \& Simon, 2012; Ropper, Samuels \& Klein, 2014). Se afirma que lo anterior sucede por la mayor densidad de receptores de dopamina, su hipersensibilidad o una función presináptica anormal (Singer \& Minzer, 2003).

Además, recientemente se ha encontrado una plasticidad mayor y anormal en la corteza motora de los pacientes con este síndrome, en comparación con los sujetos control; dicha plasticidad se encontró asociada a la severidad de los tics (Martín-Rodríguez, Ruiz-Rodríguez, Palomar, Cáceres-Redondo, Vargas, Porcacchia, Gómez-Crespo, Huertas-Fernández, Carrillo, Madruga-Garrido \& Mir, 2015).

La otra teoría que se considera de gran importancia es la neuroanatómica, en la cual evidencia respaldada empíricamente afirma anormalidades en los ganglios basales y las vías córtico-estriado-tálamo-corticales (CSTC) como la patogénesis central de los tics (Bloch \& Leckman, 2008; Greenberg, Aminoff \& Simon, 2012), pues estas vías pueden ser vistas como una estación de paso entre la intención y la acción.

Es importante mencionar que el $90 \%$ de los pacientes que padecen TS, padecen de otros desórdenes psiquiátricos, es decir es un padecimiento realmente comórbido, principalmente con el trastorno obsesivo compulsivo y el déficit atencional e hiperactividad (Ganos et al., 2013).

Los tics además son presentidos con anterioridad por los pacientes (conocidos como impulsos premonitorios), de modo que esto produce tensión, sensaciones corporales displacenteras y urgencia por moverse. Si bien estos pueden ser suprimidos por segundos o hasta horas, una vez que se ejecutó el tic, las sensaciones displacenteras y la tensión es descargada y por lo tanto, desaparece dicha tensión (Ganos et al., 2013), por esta razón se fomenta el aprendizaje del tic y su próxima y cada vez más habitual repetición debido al condicionamiento por reforzamiento negativo.

De este modo, al presentar síntomas tanto motores como comportamentales y cognitivos (Cavanna \& Rickards, 2013) es un padecimiento que interfiere con el desempeño del individuo en su ambiente.

Es a partir del entrenamiento en biofeedback (BFB), que es posible realizar una retroalimentación de tipo auditiva o visual; pues esta es una terapia psicofisiológica que mide las respuestas fisiológicas como lo son el ritmo cardíaco, la respuesta galvánica de la piel y los patrones de ondas cerebrales; que son presentados en línea con un sujeto (Frank \& Eugenio, 2013).

Por su parte, la regulación de la respiración es fundamental para el buen funcionamiento psicofísico de los seres humanos, ya que el proceso respiratorio se encuentra vinculado a procesos cognitivos, emocionales y comportamentales. Con respecto a la respiración diafragmática, se afirma que esta posee una asociación psicológica con la estabilidad emocional, el sentido de control sobre el ambiente, la calma, un alto nivel de actividad mental y física y ausencia relativa de estresores percibidos (Fried, citado por Mabel, 1996). 
Por su parte la relajación muscular progresiva (RMP) consiste en buscar "un nivel de relajación general, así como un estado de autorregulación del organismo, sobre la base de la distensión de la musculatura esquelética y mediante una serie de ejercicios de tensión-distensión de los principales grupos musculares" (brazos, cabeza, tronco y piernas) (Chóliz, 2013, p.3).

A partir de esto, se hipotetiza que a medida que el participante alcanza un estado de relajación, disminuye la frecuencia de los tics. Por lo que el objetivo general del trabajo consiste en realizar un entrenamiento en RMP con BFB para lograr la disminución de la frecuencia de tics en un estudiante universitario con Síndrome de Tourette.

Con base en lo anterior se busca que el participante alcance un estado de relajación por medio de la respiración diafragmática y la RMP lo cual construirá a la disminución de los tics. Este trabajo por tanto se considera de gran aporte debido al vacío que se encuentra en el país en esta área, únicamente existe, en Costa Rica, una organización no lucrativa ASTUTA surgida en el año 2005 , que busca dar a conocer el TS y los trastornos afines en un contexto donde se desconoce al respecto. ASTUTA es una organización comprometida con el desarrollo de proyectos, acciones y programas que generen alianzas entre las diversas organizaciones buscando que los pacientes con síndrome de Tourette tengan acceso a las condiciones pertinentes con igualdad de oportunidades (ASTUTA, s.f.).

\section{Método}

El presente trabajo consistió en un tratamiento de relajación realizado a un estudiante universitario, el cual se dividió en dos fases: La primera fase consistió en enseñar al participante a realizar una respiración diafragmática. La segunda fase que consistió en añadir una RMP a la respiración diafragmática, que consiste en tensar ciertos músculos lo más que se pueda por cinco segundos para seguidamente detener la tensión, ambas fases conducen a la relajación (Cholíz, 2013).

\section{Participante}

Para la realización del trabajo se solicitó a la Asociación Costarricense de Síndrome de Tourette y Trastornos Afines (ASTUTA) la participación voluntaria de un sujeto. Se contó con la participación de un joven de veinte años de edad, estudiante de la Universidad de Costa Rica, perteneciente a la asociación y diagnosticado con el Síndrome de Tourette hace seis años. El participante no toma ninguna medicación para el Síndrome de Tourette al momento de la intervención.

\section{Instrumentos}

Equipo de BFB o retroalimentación: marca Thougth Technology, modelo Procomp2, incluye la galga respiratoria, la cual mide el volumen de aire contenido en los pulmones y registra la tasa respiratoria; el pletismógrafo, para recolectar la frecuencia cardíaca, ambos aparatos conectados a una computadora con el programa Biograph Infinity, el cual provee retroalimentación en forma digital, de las respuestas fisiológicas medidas en el o la participante de manera que si el participante logra sincronizar el pulso con la respiración se moverá una imagen que emite un sonido lo cual facilita el aprendizaje del paciente sobre la autorregulación de su cuerpo por medio de la motivación y el reforzador positivo que a la vez provee el equipo de BFB.

Además se utiliza la técnica de relajación muscular progresiva: que consiste diversos pasos para relajar diferentes grupos musculares, los pasos que se encuentran en el anexo 1. Y la respiración diafragmática que consiste en enseñarle al participante a relajarse por medio de una respiración controlada y regular que utiliza el diafragma. Primero el participante se sienta cómodamente y con postura correcta en una silla, colocando una mano en el pecho y la otra al término de las costillas. Seguidamente se respira por medio de la nariz, provocando que la mano en el estómago se mueva, sin que el pecho lo haga. Se debe exhalar por la boca produciendo gran movilidad en costillas y la parte superior del abdomen, de manera que el pecho siga sin moverse (Chóliz, 2013). Esta respiración reduce la ansiedad por "un incremento en la actividad parasimpática debido a la estimulación del nervio vago" (Ibídem, p. 18).

\section{Procedimiento}

Inicialmente se planteó un primer encuentro o sesión, el día 3 de octubre del año 2014 en el laboratorio de fisiología de Psicología ubicado en el tercer piso de ciencias sociales de la Universidad de Costa Rica en la sede Rodrigo Facio. En esta se conoció al participante, así como el mismo conoció a las encargadas del entrenamiento y a la supervisora del trabajo; además se obtuvo el consentimiento informado del participante para formar parte del entrenamiento. Se explicó el objetivo y la metodología del trabajo, y se le preguntaron datos personales y sociodemográficos (Anexo 2).

El entrenamiento específicamente se llevó a cabo durante cinco sesiones con sus respectivos protocolos (Anexo 3). A partir de la sesión dos, realizada el 17 de octubre del 2014, las sesiones tres y cuatro tuvieron lugar el jueves y viernes 23 y 24 de octubre respectivamente, la quinta sesión se realizó 31 de octubre y la sexta el viernes 7 de noviembre. Los jueves se llevaron a cabo en la clase 320 del edificio de la facultad de ciencias sociales de Universidad de Costa Rica, en el tercer piso a la 1:30 pm de la tarde y los viernes en la cámara de Gessel de la 
escuela de psicología, ubicada igualmente en el tercer piso de ciencias sociales a la 1:00 pm de la tarde. Cada sesión tuvo una duración aproximada de 40 minutos.

En la sesiones dos, tres y cuatro se realizó únicamente la respiración diafragmática y en la cinco y seis, la RMP.

\section{Resultados}

A lo largo de las sesiones se observó un descenso en la tasa respiratoria, ya que se obtuvo una tasa respiratoria de 9.25 en la segunda sesión y una de 9.03 en la tercera. Igualmente se observa una disminución de esta en la cuarta sesión ya que se obtiene una tasa de 7.48 respiraciones por minuto.

Con respecto a la tasa cardíaca en la segunda sesión se obtuvo una de 80.80 latidos por minutos mientras que en la sesión tres esta fue de 93.68. En la cuarta volvió a un rango de 84.98 , cifra cercana a la obtenida en la segunda sesión.

En la quinta y sexta sesión se agregó la técnica de RMP, de manera que se midieron en dos tiempos la tasa cardíaca y respiratoria del participante, una vez antes de la técnica de RMP y posteriormente a esta.

Se obtuvo por tanto en la quinta sesión, una tasa respiratoria de 7.12 antes de técnica de la RMP, mientras que después la tasa respiratoria descendió a 6.38, alcanzando lo que se considera una tasa respiratoria acorde a un estado de relajación (Sharma, 2005). La tasa cardíaca pasó de 77.88 latidos por minuto a 81.69. A pesar del aumento, que pudo deberse a la agitación del participante al realizar los ejercicios, la sincronía entre su tasa cardíaca y su tasa respiratoria fue notoriamente mejor en la segunda línea base tomada después de la RMP.

En la última sesión se observa lo inverso en la tasa respiratoria a lo que se registró en la quinta sesión; ya que esta antes de la técnica de RMP fue menor a la obtenida después, estos valores son 3.62 y 4.45 respiraciones por minuto respectivamente. Lo obtenido a la tasa cardíaca coincide con el registro de la quinta sesión ya que en ambas la tasa cardíaca aumentó después de la técnica de RMP, los valores obtenidos fueron 61.44 y 98.46 latidos por minuto respectivamente (Tabla 1 ).

Tabla 1. Progreso de tasa cardiaca y respiración por minuto en cada sesión

\begin{tabular}{|c|c|c|c|c|c|c|c|}
\hline & Sesión 2 & Sesión 3 & Sesión 4 & $\begin{array}{l}\text { Sesión } 5 \\
\text { antes de } \\
\text { RMP }\end{array}$ & $\begin{array}{l}\text { Sesión } 5 \\
\text { después } \\
\text { de RMP }\end{array}$ & $\begin{array}{l}\text { Sesión } 6 \\
\text { antes de } \\
\text { RMP }\end{array}$ & $\begin{array}{l}\text { Sesión } 6 \\
\text { después } \\
\text { de RMP }\end{array}$ \\
\hline $\begin{array}{l}\text { Tasa } \\
\text { Respiratoria }\end{array}$ & 9.25 & 9.03 & 7.48 & 7.12 & 6.38 & 3.62 & 4.45 \\
\hline $\begin{array}{l}\text { Frecuencia } \\
\text { cardiaca }\end{array}$ & 80.80 & 93.68 & 84.98 & 77.88 & 81.69 & 61.44 & 98.46 \\
\hline
\end{tabular}

Sobre los tics, se contabilizaron treinta en la segunda sesión (Anexo 4) en total mientras que en la tercera se contabilizaron veintitrés y en la cuarta dieciséis en total. Sin embargo este descenso no continúa en la quinta sesión, ya que el tiempo de la sesión y así mismo el tiempo de contabilización de tics, aumentaron ya que se añadió la técnica de RMP, de manera que las sesiones pasaron de veinte minutos a cuarenta minutos en la quinta y cincuenta minutos en la sexta sesión.

De esa forma se contabilizaron veinte tics en total en la sesión cinco (Anexo 7) y veintiséis en la sesión 6 (Anexo 8).

Se observa con respecto a la tasa respiratoria que hubo un descenso ya que en la segunda sesión esta fue de de 9.25 y en la sexta, promediando ambos momentos fue de 4.03 (Figura 1). Con respecto a la tasa cardíaca se registran 80.80 latidos por minuto en la primera sesión a 79.95 en promedio en la última sesión (Figura 2).

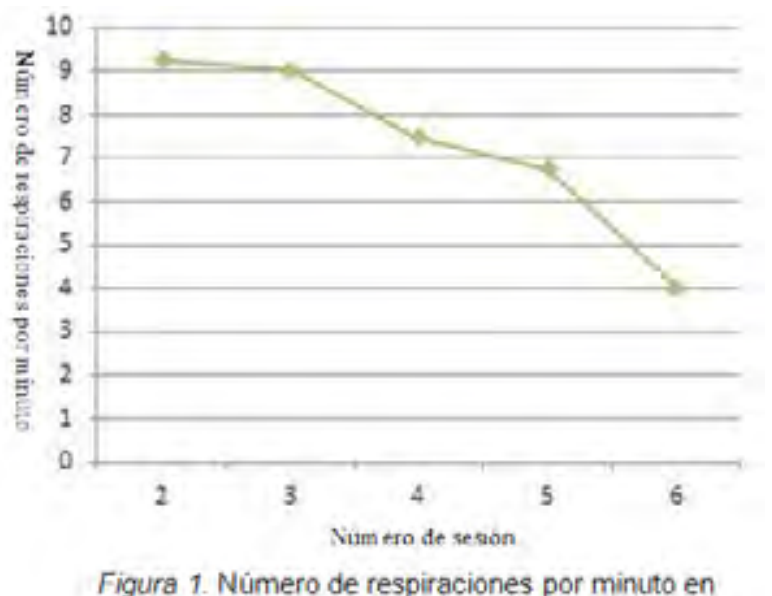

Figura 1. Nümero de respiraciones por minuto en cada una de las sesiones

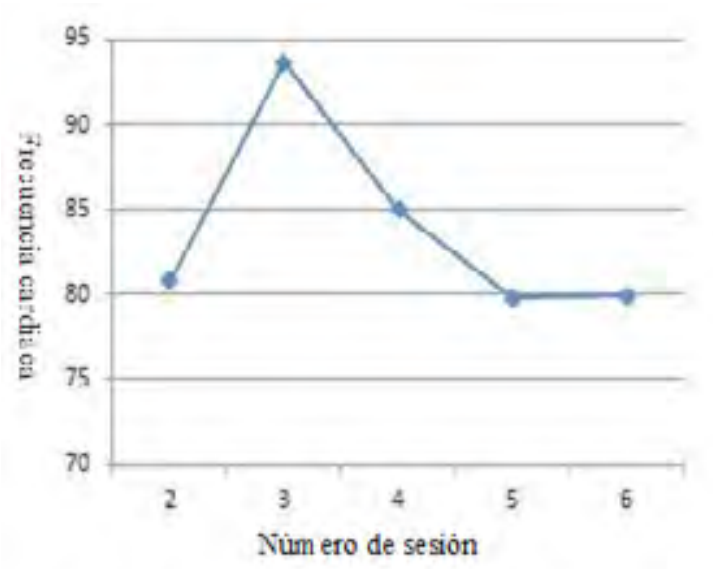

Figura 2. Frecuencia cardiaca en cada una de las sesiones 
En la tabla 2 se muestran los porcentajes respecto a cada sesión y con su respectivo tiempo. Tomando la segunda sesión como base, ya que es la que muestra mayor cantidad de tics por minuto, se puede observar que porcentualmente en la tercera sesión hubo un $24 \%$ menos de tics a lo largo de la sesión, en la cuarta sesión hubo un $46,67 \%$ menos cantidad de tics en comparación a la segunda sesión. Para la quinta sesión los tics disminuyen en un $66,67 \%$ llegando a su punto más bajo, y para la sexta y última sesión, se redujeron en un $65,36 \%$.

Por lo anterior, se puede evidenciar que los tics se redujeron en más de un $50 \%$ al final de las sesiones.

Tabla 2. Porcentaje de tics en cada sesión

\begin{tabular}{lllll}
\hline Sesión & Tiempo (minutos) & Cantidad de Tics & Tics por minuto & Porcentaje (\%) \\
\hline 2 & 20 & 30 & 1,5 & 100 \\
3 & 20 & 23 & 1,15 & 76 \\
4 & 20 & 16 & 0,8 & 53,33 \\
5 & 40 & 20 & 0,5 & 33,33 \\
6 & 50 & 26 & 0,52 & 34,66
\end{tabular}

\section{Discusión}

A través de la realización de este trabajo, se puede observar que se dio una disminución cuantitativa de los tics conforme avanzaron las sesiones, lo cual obtuvo una concordancia con la disminución de la frecuencia cardiaca en el participante. A raíz de esto, parece existir evidencia que apoya nuestra hipótesis inicial, en cuanto a que los tics podrían estar relacionados con la ansiedad. Conforme el participante muestra un mayor estado de relajación, se da una disminución total de los tics, respaldando lo afirmado por las investigaciones previas sobre el tema y mencionadas anteriormente.

En los resultados obtenidos, se puede observar porcentualmente en el transcurso de las sesiones, en donde la cantidad de los tics que presentaba el paciente fue disminuyendo. En la tercera sesión hubo un $24 \%$ menos de tics que en la segunda sesión, en la cuarta sesión hubo un $46,67 \%$ menos cantidad de tics en comparación a la segunda sesión. Para la quinta sesión los tics disminuyeron en un $66,67 \%$ llegando a su punto más bajo, y para la última sesión, la sexta, se redujeron en un $65,36 \%$; esto evidencia que los tics se redujeron de manera generalizada en más de un $50 \%$. Esto apoya la hipótesis planteada inicialmente.

Uno de los resultados más importantes que se pudieron obtener fue el hecho de que en la tercera sesión, los tics que presentaron mayor frecuencia fueron los de tipo vocales y movimientos de las piernas, con seis y cinco repeticiones respectivamente. Sin embargo, en el transcurso de las sesiones, en la cuatro, cinco y seis, se presentaron los porcentajes más bajos de frecuencia, con una totalidad nula de tics en estas partes.

Con respecto a la tasa cardíaca, en algunas sesiones se observó un aumento de este después de la realización de ejercicios de tensión muscular, lo cual se puede deber a esta misma actividad física de la RMP que demanda cierta fuerza emitida por el participante.

Se pudo observar que la tasa respiratoria disminuyó significativamente al momento de aplicar la técnica de relajación muscular lo que permitió al individuo llegar su estado máximo de relajación en las sesiones. Lo anterior entonces logró el objetivo principal de esta técnica, la cual Chóliz (2013), afirma que permite un estado de relajación y de autorregulación del individuo; estado que como ya se ha mencionado, se ha demostrado que el aumento de la tensión y la ansiedad provocan un aumento de los mismos (Conelea, Woods \& Brandt, 2011; Felling \& Singer, 2011; Busea, Schoenefelda, Münchaub \& Roessnera, 2013; Frank \& Cavanna, 2013; Neuner, et al. 2014).

Por otro lado, el hecho de que se presentaran una mayor cantidad de tics en la cuarta sesión que en la segunda, se explica por el hecho de que los resultados se obtuvieron por el número de tics presentados por minuto. Sin embargo, tomando en cuenta que la quinta sesión duró veinte minutos más que la segunda sesión, se puede ver un porcentaje equitativo; lo cual se comprobó en el 50\% de la reducción final de los tics durante la terapia de relajación.

A partir de esto, la presente investigación parece haber tenido un impacto en el establecimiento de la relajación, favoreciéndola, lo cual se demostró en una disminución tanto en los tics, como de la tasa cardíaca y la tasa respiratoria. Lo anterior se denotó principalmente en el hecho del aumento de la frecuencia de los tics en momentos donde se establecían las instrucciones de la tarea, mientras que se disminuyeron en la respiración diafragmática y se dejaron de presentar en los momentos de relajación muscular.

En la misma línea, el participante afirmó que sentía una diferencia cualitativa y cuantitativa en el cambio de las sesiones de relajación, pues consideró que se dio una reducción y un control mayor de los tics en las sesiones de RMP, así como también un mayor provecho de las mismas por el tiempo de tratamiento. A partir de esto, se puede justificar la razón por la cual se denotó una mayor sincronía en la tasa cardiaca y en la tasa respiratoria de la línea base obtenida después de la relajación muscular, lo que permitió que el participante lograra una autoconsciencia corporal, que permite controlar sus tics de forma autónoma e independiente. Sin embargo por el esfuerzo mencionado anteriormente que demanda la RMP, se recomienda para este padecimiento, solo dedicarse a la respiración e ir enseñándole la RMP y hasta que él haya generalizado la sensación y logre ambos ejercicios, someterlo al BFB, pero ya sin realizar los movimientos.

Finalmente, es importante considerar el hecho de que el participante afirmó sentir una diferencia a nivel físico cuando se encuentra en un estado de 
relajación, estado que según él mismo ha ayudado a lograr una reducción de los tics por medio de la coordinación del ritmo cardíaco y la respiración; lo cual lleva a una relajación en sus actividades de vida cotidiana independientes a la terapia realizada por las investigadoras.

Lo anterior entonces es un hecho de gran relevancia para la psicología en temas de autosuficiencia y autoconsciencia de las propias acciones, de formas de comportamiento y de control del mismo, lo que permite al individuo una modificación de las relaciones con el mundo exterior.

\section{Referencias}

American Psychiatric Association. (2013). Diagnostic and statistical manual of mental disorders (5th ed.). Arlington, VA: American Psychiatric Publishing.

ASTUTA (s.f.). Asociación Costarricense de Síndrome de Tourette y Trastornos Afines. Recuperado de: http://www.sindromedetourette.com/

Bloch M.H., Leckman J.F. (2008). Chapter 44. Tourette Disorder and ObsessiveCompulsive Disorder in Children and Adolescents. In Ebert M.H., Loosen P.T., Nurcombe B, Leckman J.F., CURRENT Diagnosis \& Treatment: Psychiatry, 2e.New York: McGraw-Hill.

Busea, J., Schoenefeld, K., Münchau, A. \& Roessner, V. (2013). Neuromodulation in Tourette syndrome: Dopamine and beyond. Neuroscience and Biobehavioral Reviews, 37, 1069-1084.

Carlson, N. (2013). Physiology of Behavior. New Jersey: Pearson Education, Inc.

Cavanna, A. \& Rickards, H. (2013). The psychopathological spectrum of Gilles de la Tourette syndrome. Neuroscience and Biobehavioral Reviews, 37, 10081015.

Chóliz, M. (2013).Técnicas para el control de la activación: Relajación y respiración. Valencia: Universidad de Valencia.

Conelea, C., Woods, D. \& Brandt, B. (2011). The impact of a stress induction task on tic frequencies in youth with Tourette Syndrome. Behaviour Research and Therapy, 49, 492-497.

Crossley, E., Seri, S., Stern, J., Robertson, M. \& Cavanna, A. (2014). Premonitory urges for tics in adult patients with Tourette Syndrome. Brain \& Development, $36,45-50$.
Denys, D., de Vriesc, F., Cathe, D., Figeea, M., Vulinka, N., Veltmana, D., van der Doef, T., Boellaardd, R., Westenbergf, H., Balkomc, A., Lammertsmad, A. \& van Berckeld, B. (2013). Dopaminergic activity in Tourette syndrome and obsessive-compulsive disorder. European Neuropsychopharmacology, 23, $1423-1431$.

Felling \& Singer (2011). Neurobiology of Tourette Syndrome: Current Status and Need for Further Investigation. The Journal of Neuroscience, 31 (35), 2387-12395.

Frank, M. \& Eugenio, A. (2013). Behavioral treatments for Tourette syndrome: A evidence based - review. Behavioral Neurology, (27), 105-117.

Ganos, C., Roessner, V. \& Münchau, A. (2013). The functional anatomy of Gilles de la Tourette syndrome. Neuroscience and Biobehavioral Reviews, 37, 10501062.

Greenberg D.A., Aminoff M.J., Simon R.P. (2012). Chapter 11. Movement Disorders. In Greenberg D.A., Aminoff M.J., Simon R.P. (Eds), Clinical Neurology, 8e. New York: McGraw-Hill.

Martín-Rodríguez, J., Ruiz-Rodríguez, M., Palomar, F., Cáceres-Redondo, M., Vargas, L., Porcacchia, P., Gómez-Crespo, M., Huertas-Fernández, I., Carrillo, F., Madruga-Garrido, M. \& Mir, P. (2015). Aberrant cortical associative plasticity associated with severe adult Tourette syndrome. Mov Disord, 30 (3), 431-5.

Longo, D., Fauci, A., Kasper, D., Hauser, S., Jameson J. \& Loscalzo, J. (2011). Harrison's Principles of Internal Medicine (18th Edition). Boston: McGraw Hill Professional.

Mabel, L. (1996). La técnica de la respiración: Aportes psicoterapéuticos. Salud Mental, 19 (4), 31-35.

Mermillod, M., Vermeulen, N., Droit-Volet, S., Jalenques, S., Durif, F. \& Niedenthal, P. (2011). Embodying Emotional Disorders: New Hypotheses about Possible Emotional Consequences of Motor Disorders in Parkinson's Disease and Tourette's Syndrome. ISRN Neurology, Special Section, 1-6.

Middaugh, S. J., Woods, S. E., Kee, W.G., Harden, R. N. \& Peters, J. R. (1991). Biofeeedback asssisted relaxation training for aging chronic pain patient. Biofeedback and Self-regulation,16(4), 361-377.

Neuner, I., Cornelius, J.W., Arrubla, J., Stocker, T., Ehlen, C., Wegner, H.D., Schneider, F., Shah, N.J. (2014). Imaging the where and when of tic generation and resting state networks in adult Tourette patients. Neuroimage, 362, 1-16. 
Serajee, F. \& Mahbubu, A. (2015). Advances in Tourette Syndrome: Diagnoses and Treatment. Pediatr Clin N Am, 62, 687-701.

Sharma, V. (2005). Breath is the key to wellness. Mind Publications, s.p.

Singer, H.S., Minzer, K. (2003) Neurobiology of Tourette syndrome: concepts of neuroanatomical localization and neurochemical abnormalities. Brain Dev, 25 (Suppl), S70-S84

\section{Anexos}

\section{Anexo 1: Técnica de relajación progresiva}

1 y 3.- Apretar el puño, contrayendo mano, muñeca y antebrazo.

2 y 4.- Contraer el biceps empujando el codo contra el respaldo del sillón o contra el colchón.

5. Frente: elevar las cejas o arrugar la frente

6. Parte superior cara y nariz: arrugar la nariz y los labios

7. Parte inferior de la cara y mandíbulas: apretar los dientes y hacer una

sonrisa forzada tirando de la comisura de los labios hacia afuera.

8. Pecho y garganta: empujar la barbilla como hacia abajo como si quisiera que tocara el pecho, pero al mismo tiempo hacer fuerza para que no lo consiga, es decir, contraponer los músculos frontales y posteriores del cuello. 9. Hombros y espalda. A la vez que inspira arquee la espalda como si quisiera unir los omoplatos entre sí.

10.- Abdomen. Poner el estómago duro y tenso, como si quisiera contrarrestar un fuerte golpe.

11 y 14.- Piernas: colocar la pierna estirada y subirla hacia arriba $20 \mathrm{cms}$, tensando y haciendo fuerza como si tuviera un peso en el pie, que debiera sujetar a una altura de $20 \mathrm{cms}$. Para relajar soltar la pierna y que caiga a peso.

$12,13,15$ y 16 . Ejercicios de pantorrilla y pie. Existen dos movimientos: a) estirar la pierna y la punta de los dedos como si quisiera tocar un objeto delante

b) doblar los dedos hacia atrás como si quisiera tocarse la rodilla con ellos. Es importante tener en cuenta que estos dos últimos ejercicios de $h$ debe hacerlos SIN FORZAR. La tensión debe ser suave, es suficiente para relajar las pantorrillas y los pies sin producir ningún daño.

Fuente: Chóliz, 2013, p. 4 


\section{Anexo 2: Entrevista al participante}

\section{Nombre Completo:}

Sexo: masculino

Peso: 65 kilos (aproximadamente)

Edad: 20

Ocupación: Estudiante de terapia física

Estado civil: Soltero

Edad en que notaron la aparición del síndrome

Seis, probablemente antes, pero no sé

Edad en que fue diagnosticado con el síndrome:

Seis

¿Ha utilizado algún medicamento?

Antes de cuarto grado. Ritalina y risperdal.

¿Realiza alguna actividad física, Cual?

Sí. Karate, judo y pesas; pesas todavía en clases de vez en cuando.

Actividades en el tiempo libre

Deporte, más que todo.

Experiencia con el padecimiento de tourette

(Se ríe)

- No sé.

-No me molesta.

- No interviene.

Anexo 3: Machote del protocolo realizado para cada sesión

\begin{tabular}{llllll}
\hline Objetivo & Metas & Indicador & Materiales & Rol del entrenador & Tiempo \\
\hline
\end{tabular}

Anexo 4: Contabilización de tics en la segunda sesión

\begin{tabular}{ll}
\hline Tipo de tics & Cantidad de tics \\
\hline Motores faciales & 4 \\
\hline Vocales & 6 \\
\hline Pestañeos & 7 \\
\hline Movimiento de cabeza & 5 \\
\hline Movimiento de piernas & 5 \\
\hline Movimiento de hombros & 3 \\
\hline Total & 30 \\
\hline
\end{tabular}

Anexo 5: Contabilización de tics en la tercera sesión

\begin{tabular}{ll}
\hline Tipo de tics & Cantidad de tics \\
\hline Motores faciales & 3 \\
\hline Vocales & 6 \\
\hline Pestañeos & 3 \\
\hline Movimiento de cabeza & 5 \\
\hline Movimiento de piernas & 5 \\
\hline Movimiento de hombros & 1 \\
\hline Total & 23 \\
\hline
\end{tabular}

Anexo 6: Contabilización de tics en la cuarta sesión

\begin{tabular}{ll}
\hline Tipo de Tics & Cantidad \\
\hline Motores faciales & 1 \\
\hline Vocales & 0 \\
\hline Pestañeos & 9 \\
\hline Movimiento de cabeza & 2 \\
\hline Movimiento de piernas & 0 \\
\hline Manos & 1 \\
\hline Movimiento de hombros & 3 \\
\hline Total & 16 \\
\hline
\end{tabular}

Anexo 7: Contabilización de tics en la quinta sesión

\begin{tabular}{ll}
\hline Tipo de Tics & Cantidad \\
\hline Motores faciales & 1 \\
\hline Vocales & 0 \\
\hline Pestañeos & 7 \\
\hline Movimiento de cabeza & 8 \\
\hline Movimiento de piernas & 0 \\
\hline Manos & 0 \\
\hline Movimiento de hombros & 4 \\
\hline Total & 20
\end{tabular}


24 Wímblu, Rev. estudiantes Esc. de psicología, Univ. Costa Rica. 12 (1) (marzo-agosto): 9-24, 2017 / ISSN:1659-2107

Anexo 8: Contabilización de tics en la sexta sesión

\begin{tabular}{ll}
\hline Tipo de Tics & Cantidad de Tics \\
\hline Motores facials & 3 \\
\hline Vocales & 0 \\
\hline Pestañeos & 15 \\
\hline Movimiento de Cabeza & 2 \\
\hline Movimiento de piernas & 0 \\
\hline Manos & 0 \\
\hline Movimiento de hombros & 6 \\
\hline Total & 26 \\
\hline
\end{tabular}

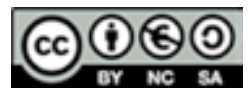

Esta obra está bajo una licencia de Creative Commons Reconocimiento

- NoComercial - CompartirIgual (by-nc-sa) 